Phys. Rev. B (01Aug99)

\title{
Neutron-scattering study of spin-density wave order in the superconducting state of excess-oxygen-doped $\mathrm{La}_{2} \mathrm{CuO}_{4+y}$
}

\author{
Y. S. Lee, R. J. Birgeneau, and M. A. Kastner \\ Department of Physics and Center for Materials Science and Engineering, Massachusetts Institute of Technology, Cambridge, \\ Massachusetts 02139 \\ Y. Endoh and S. Wakimoto \\ Department of Physics, Tohoku University, Aramaki Aoba, Sendai 980-77, Japan \\ K. Yamada \\ Institute for Chemical Research, Kyoto University, Gokasho, Uji 610-0011, Japan \\ R. W. Erwin and S.-H. Lee \\ National Institute of Standards and Technology, Gaithersburg, Maryland 20899 \\ G. Shirane \\ Department of Physics, Brookhaven National Laboratory, Upton, New York 11973
}

(June 30, 2021)

\begin{abstract}
We report neutron scattering measurements of spin density wave order within the superconducting state of a single crystal of predominately stage- $4 \mathrm{La}_{2} \mathrm{CuO}_{4+y}$ with a $T_{c}$ (onset) of $42 \mathrm{~K}$. The low temperature elastic magnetic scattering is incommensurate with the lattice and is characterized by long-range order in the copper-oxide plane with the spin direction identical to that in the insulator. Between neighboring planes, the spins exhibit short-range correlations with a stacking arrangement reminiscent of that in the undoped antiferromagnetic insulator. The elastic magnetic peak intensity appears at the same temperature within the errors as the superconductivity, suggesting that the two phenomena are strongly correlated. These observations directly reveal the persistent influence of the antiferromagnetic order as the doping level increases from the insulator to the superconductor. In addition, our results confirm that spin density wave order for incommensurabilities near $1 / 8$ is a robust feature of the $\mathrm{La}_{2} \mathrm{CuO}_{4}$-based superconductors.
\end{abstract}

PACS numbers: 74.72.Dn, 75.10.Jm, 75.30.Fv, 75.50.Ee

\section{INTRODUCTION}

In the copper-oxide superconductors, the multiple roles played by the electrons continue to defy a comprehensive theoretical understanding. As carriers of both spin and charge, the strongly interacting electrons are responsible for both the unconventional microscopic magnetic behavior and the unusual macroscopic electronic properties of the doped cuprates. Localized antiferromagnetism is a dominant feature of the cuprate phase diagram at low doping levels, while conventional itinerantelectron behavior appears to obtain in the overdoped, non-superconducting regime. The intermediate doping levels are dominated by neither, and this is where hightemperature superconductivity occurs. Over the past decade, experimental probes such as nuclear magnetic resonance (NMR), muon spin resonance ( $\mu \mathrm{SR})$, and neutron scattering have revealed a fascinating interplay between the spin fluctuations and the superconductivity in the lamellar copper-oxides. In particular, neutron scattering experiments have conclusively shown that dynamic antiferromagnetic correlations are a robust feature of the suprates in both the superconducting and normal states.t.

Additionally, it is becoming increasingly apparent that incommensurate spin fluctuations are universal to the high $T_{c}$ superconductors. The low-energy magnetic excitations in $\mathrm{La}_{2-x} \mathrm{Sr}_{x} \mathrm{CuO}_{4+y}$ superconductors have been extensively studied, and the observed spin fluctuations are characterized by wayfvectors which are incommensurate with the lattice.2. These modulated spin fluctuations persist in both the normal and superconducting states, with a suppression of their intensity occurring below $T_{c}$. Yamada and coworkers have shown in $\mathrm{La}_{2-x} \mathrm{Sr}_{x} \mathrm{CuO}_{4}$ that the incommensurability $\delta$ increases progressively with the doping level for $x \geq 0.05$ with the simple empirical relation $\delta \simeq x$, but saturates near a value of $\delta \simeq 1 / 8$ above a doping level of $x \simeq 1 / 8$. Recently, incommensurate magnetic scattering has been seen in superconducting $\mathrm{YBa}_{2} \mathrm{Cu}_{3} \mathrm{O}_{6+x}$ with the explicit incompnensurabilities consistent with those in $\mathrm{La}_{2-x} \mathrm{Sr}_{x} \mathrm{CuO}_{4} \mathrm{~d}$ There still exist several competing models to explain the spatially modulated spin correlations, with plausible explanations ranging from spin-flip 
electron scattering across the Fermi surfaced 9 to fluctuating stripes of antiferromagnetic spins in amiroscopically phase-separated doped Mott insulator 1012.

Much recent interest has focused on the observation of static incommensurate magnetic ordering which coexists with superconductivity in certain $\mathrm{La}_{2} \mathrm{CuO}_{4}$ based systems. In $\mathrm{La}_{1.6-x} \mathrm{Nd}_{0.4} \mathrm{Sr}_{x} \mathrm{CuO}_{4}$, Tranquada and coworkers 13 have found evidence for a static ordering of charge and spin with an incommensurate modulation. They propose a picture of antiferromagnetically ordered stripe regions separated by charged domain walls which act as magnetic antiphase boundaries. 10.13 The three $\mathrm{La}_{1.6-x} \mathrm{Nd}_{0.4} \mathrm{Sr}_{x} \mathrm{CuO}_{4}$ samples studied with $x=0.12$, 0.15 , and 0.20 all exhibit elastic incommensurate magnetic peaks with onset temperatures of $\simeq 50 \mathrm{~K}, 44 \mathrm{~K}$, and $15 \mathrm{~K}$, respectively. For the $x=0.12$ sample there is also evidence for charge ordering with a somewhat higher onset temperature. The incommensurabilities of the static magnetic order are consistent with the dynamic spin fluctuation incommensurabilities measured by Yamada et al. 2 in samples of $\mathrm{La}_{2-x} \mathrm{Sr}_{x} \mathrm{Cu}_{4}$, that is, without $\mathrm{Nd}^{3+}$ co-doping. Subsequent work 14 has shown that in the $x=0.12$ sample, $\mathrm{La}_{1.48} \mathrm{Nd}_{0.4} \mathrm{Sr}_{0.12} \mathrm{CuO}_{4}$, the correlation length reaches its maximum value of $\sim 200 \AA$ below $\sim 30 \mathrm{~K}$. The $x=0.12,0.15,0.20$ samples are all superconducting with onset $T_{c}$ 's of $\simeq 4 \mathrm{~K}, 11 \mathrm{~K}$, and $15 \mathrm{~K}$, respectively; here each $T_{c}$ is reduced with respect to its value in the absence of $\mathrm{Nd}^{3+}$ co-doping. This reduction of the superconducting $T_{c}$ 's in $\mathrm{La}_{1.6-x} \mathrm{Nd}_{0.4} \mathrm{Sr}_{x} \mathrm{CuO}_{4}$ is believed to have the same origin as the suppressed $\mathrm{s} H$ perconductivity in $\mathrm{La}_{2-x} \mathrm{Ba}_{x} \mathrm{CuO}_{4}$ with $x$ near 0.125.15 Both systems are tetragonal below $\sim 70 \mathrm{~K}$, in contrast to $\mathrm{La}_{2-x} \mathrm{Sr}_{x} \mathrm{CuO}_{4}$ which retains the orthorhombic structure down to the lowest temperatures measured. Noting that the $\mathrm{La}_{1.6-x} \mathrm{Nd}_{0.4} \mathrm{Sr}_{x} \mathrm{CuO}_{4}$ samples with the largest magnetic order parameters also have the lowest superconducting $T_{c}$ 's, Tranquada et al. posit that the static stripe ordering and the superconductivity compete with each other. In addition, they speculate that the low temperature tetragonal (LTT) phase is essential for the static magnetic order in the superconductors. Indeed, $\mu \mathrm{SR}$ experiments have detected the reemergence of magnetic ordering in $\mathrm{La}_{2-x} \mathrm{Ba}_{x} \mathrm{CuO}_{4}$ and $\mathrm{La}_{1.6-x} \mathrm{Nd}_{0.4} \mathrm{Sr}_{x} \mathrm{CuO}_{4}$ samples at doping levels for which the superconductivity is suppressed and the low temperature structure is tetragonal.16.17

Experiments on $\mathrm{La}_{2-x} \mathrm{Sr}_{x} \mathrm{CuO}_{4}$ show, however, that the existence of the LTT structural phase is not a necessary condition for the appearance of static magnetic order. Based on their NMR studies of $\mathrm{La}_{2-x} \mathrm{Sr}_{x} \mathrm{CuO}_{4}$ $(x \sim 0.115)$ and $\mathrm{La}_{2-x} \mathrm{Ba}_{x} \mathrm{CuO}_{4}(x \simeq 0.125)$, Goto et al. 18 conclude that magnetic order exists even in the absence of the LTT structure. More direct evidence has come from recent neutron scattering experiments on $\mathrm{La}_{2-x} \mathrm{Sr}_{x} \mathrm{CuO}_{4}$ single crystals with $x=0.10,0.12,0.13$, all of which have the low temperature orthorhombic (LTO) structure.19 21 These measurements have confirmed that incommensurate spin density wave (SDW) order exists at doping levels near $x=1 / 8$ even in the absence of $\mathrm{Nd}^{3+}$ co-doping. In particular, the work of Kimura et al.20 shows that the most dramatic effects occur for the $x=0.12$ sample, for which the elastic peak width in their measurement is resolution-limited, indicating a static magnetic correlation length exceeding $200 \AA$. Surprisingly, $T_{c}$ (onset) and the SDW ordering temperature, $T_{m}$, coincide at $\sim 31 \mathrm{~K}$ within the errors, in contrast to $\mathrm{La}_{1.48} \mathrm{Nd}_{0.4} \mathrm{Sr}_{0.12} \mathrm{CuO}_{4}$, in which $T_{m}$ and $T_{c}$ are well separated. It should be noted that, similar to the previously mentioned compounds, there is evidence that the superconductivity is sypnressed somewhat in $\mathrm{La}_{2-x} \mathrm{Sr}_{x} \mathrm{CuO}_{4}$ for $x$ near 0.115 , 182223 though not to the same extent as in $\mathrm{La}_{2-x} \mathrm{Ba}_{x} \mathrm{CuO}_{4}$ for $x \simeq 0.125$.

In view of the aformentioned studies, a fundamental issue needing further clarification is the universality of the relationship between the superconductivity, including any possible suppression of $T_{c}$, and the SDW ordering. Although it is now clear that the LTT phase is not essential for the establishment of the SDW, more experiments are necessary to search for magnetic ordering in superconducting samples which are orthorhombic rather than tetragonal. Additionally, further details of the spin structure of the magnetic order are essential to elucidate the basic physics of these systems and to test the specifics of competing models. Most importantly, such studies may shed light on the fundamental question of the appropriateness of an itinerant versus doped Mott insulator model for the superconducting lamellar copper oxides. To address these issues, we have investigated a superconducting single crystal of excess-oxygen-doped $\mathrm{La}_{2} \mathrm{CuO}_{4+y}$. For the $\mathrm{La}_{2} \mathrm{CuO}_{4}$-based superconductors, those doped with oxygen achieve the highest superconducting $T_{c}$ 's (up to $45 \mathrm{~K}$ ) and are believed to possess a relatively small degree of dopant disorder. 3 The dopant oxygen ions are mobile down to temperatures near $\sim 200 \mathrm{~K}$. Therefore, the oxygen interstitials may find their equilibrium configuration and are annealed with respect to the other major energetics of the system, at least at $200 \mathrm{~K}$; here, the magnetic exchange energy is $J \simeq 1500 \mathrm{~K}$ and the electronic bandwidth is $W \simeq 2 J$.

In this paper, we present elastic and inelastic neutron scattering data on a single crystal of $\mathrm{La}_{2} \mathrm{CuO}_{4+y}$ with a superconducting transition temperature onset of $42 \mathrm{~K}$. As we shall show, this crystal exhibits a transition to incommensurate magnetic long-range order at a temperature $T_{m}$ coincident within the uncertainties with $T_{c}$ (onset) $\sim 42 \mathrm{~K}$. We discuss in some detail the associated magnetic structure. The format of this paper is as follows: Section 2 contains preliminary details about our measurements and about the characterization of our $\mathrm{La}_{2} \mathrm{CuO}_{4+y}$ sample. The staging behavior is discussed in Section 3. The results of the inelastic magnetic neutron scattering experiments are described in Section 4. Section 5 reports elastic neutron scattering studies of the spin density wave ordering. Finally, Section 6 contains a discussion of the results, especially in the context of previous work. 


\section{PRELIMINARY DETAILS}

In contrast to NMR and $\mu \mathrm{SR}$ techniques, which are local probes, neutron scattering experiments uniquely measure the collective behavior of spins or nuclei in a crystal. The magnetic scattering cross section of localized spins for unpolarized neutrons is directly related to the Fourier transform of the space- and time-dependent spin-pair correlation function. Defining the neutron energy and momentum transfers to the spin system as $\omega=E_{f}-E_{i}$ and $\mathbf{Q}=\mathbf{k}_{\mathbf{f}}-\mathbf{k}_{\mathbf{i}}$, respectively, (here, we use units in which $\left.\hbar=k_{B}=1\right)$ the inelastic neutron cross section is given by

$$
\frac{d^{2} \sigma}{d \Omega d E} \sim f^{2}(\mathbf{Q}) \frac{k_{f}}{k_{i}} \sum_{\alpha, \beta}\left(\delta_{\alpha \beta}-\hat{Q}_{\alpha} \hat{Q}_{\beta}\right) S^{\alpha \beta}(\mathbf{Q}, \omega),
$$

where $\alpha, \beta=x, y, z, f(\mathbf{Q})$ is the magnetic form factor, and the dynamic structure factor is defined as

$$
S^{\alpha \beta}(\mathbf{Q}, \omega)=\frac{1}{2 \pi} \sum_{\mathbf{r}} \int_{0}^{\infty} d t e^{(i \mathbf{Q} \cdot \mathbf{r}-\omega t)}\left\langle S^{\alpha}(0,0) S^{\beta}(\mathbf{r}, t)\right\rangle .
$$

In ordered structures, for which the thermodynamic average of the spin density appears at each lattice site, some portion of the scattering occurs in elastic Bragg peaks. The magnetic cross section for this coherent elastic scattering is

$$
\begin{aligned}
\left.\frac{d^{2} \sigma}{d \Omega d E}\right|_{\text {Bragg }} \sim & f^{2}(\mathbf{Q}) \sum_{\alpha, \beta}\left(\delta_{\alpha \beta}-\hat{Q}_{\alpha} \hat{Q}_{\beta}\right) \\
& \times \sum_{\mathbf{d}} e^{i \mathbf{Q} \cdot \mathbf{d}}\left\langle S^{\alpha}(0)\right\rangle\left\langle S^{\beta}(\mathbf{d})\right\rangle \delta(\omega)
\end{aligned}
$$

with $\mathbf{Q}=\mathbf{G}$, a magnetic reciprocal lattice vector, and $\sum_{\mathbf{d}}$ is a sum over the ions in the magnetic unit cell. The geometrical factor $\left(\delta_{\alpha \beta}-\hat{Q}_{\alpha} \hat{Q}_{\beta}\right)$ in both cross sections is a consequence of the dipolar interaction of the neutron with the spin and allows one to determine the spin direction of static and fluctuating spins by systematically varying the momentum transfer $\mathbf{Q}$. The imaginary part of the generalized susceptibility is related to the measured dynamic structure factor via the fluctuation-dissipation theorem,

$$
\chi^{\prime \prime}(\mathbf{Q}, \omega) \frac{1}{1-e^{-\omega / T}}=S(\mathbf{Q}, \omega) .
$$

In the case of an itinerant electron system, the measured structure factor is related to the particle-hole excitations,

$$
\begin{aligned}
\chi^{\prime \prime}(\mathbf{Q}, \omega)=\sum_{\mathbf{k}} & \left(\left\langle n_{\mathbf{k} \downarrow}\right\rangle-\left\langle n_{\mathbf{k}+\mathbf{Q} \uparrow}\right\rangle\right) \\
& \times \delta[E(\mathbf{k}+\mathbf{Q})-E(\mathbf{k})-\omega] .
\end{aligned}
$$

The neutron scattering experiments described in this paper were performed on the SPINS and BT9 triple-axis spectrometers located at the NIST Center for Neutron Research in Gaithersburg, MD, and also on the TOPAN triple-axis spectrometer located at the JAERI JRR-3M reactor in Tokai, Japan. Pyrolytic graphite (PG) crystals were used to monochromate and analyze the neutron energies. For the measurements on the SPINS spectrometer, cold neutrons with an incident energy of $5 \mathrm{meV}$ were selected, and a liquid-nitrogen cooled beryllium filter was placed in the beam path to remove contamination from higher order neutron energies. For the measurements on the BT9 and TOPAN spectrometers, thermal neutrons with incident energies of $13.7 \mathrm{meV}$ or $14.7 \mathrm{meV}$ were selected, and a PG filter was placed in the incident beam to remove higher order neutron energies. The sample was sealed in an aluminum container in a He gas environment for thermal exchange. A pumped ${ }^{4} \mathrm{He}$ cryostat was used to control temperature from $1.4 \mathrm{~K}$ to $300 \mathrm{~K}$.

We have, over the past few years, developed a reproducible method for preparing single crystals of $\mathrm{La}_{2} \mathrm{CuO}_{4+y}$ that are quite large, of high purity, and with homogenous oxygen doping. First, a crystal of $\mathrm{La}_{2} \mathrm{CuO}_{4}$ is grown by the traveling solvent floating-zone method; the crystal studied here has a volume of about $0.6 \mathrm{~cm}^{3}$ with a crystal mosaic of $0.2^{\circ}$ half-width at half-maximum. In order to incorporate a large quantity of excess oxygen into the crystal, we employ an electrochemical doping technique. Previous studies on ceramic and small single crystal samples of $\mathrm{L}_{2} \mathrm{CuO}_{4+y}$ have shown that electrochemical oxidation 24 can produce higher values of $y$ than methods involving annealing in high oxygen pressure 28. Recently, we have refined the technique so that large single crystals can be homogeneously doped, and thus are suitable for magnetic neutron scattering measurements, which require large scattering volumes. Wells et al. 3 have investigated the phase diagram of $\mathrm{La}_{2} \mathrm{CuO}_{4+y}$ crystals and have reported neutron scattering measurements of the low-energy magnetic excitations in superconducting $\mathrm{La}_{2} \mathrm{CuO}_{4+y}$. The superconducting crystals examined by these authors have $T_{c}$ 's of $31-32 \mathrm{~K}$ and represent the phase on the oxygen-rich boundary of the first miscibility gap with an oxygen content of $y \simeq 0.055$.

For the present studies we have successfully oxygenated a large single crystal with an oxygen concentration well beyond the first miscibility gap. The bulk magnetic susceptibility of the $\mathrm{La}_{2} \mathrm{CuO}_{4+y}$ crystal has been measured with a SQUID magnetometer after cooling in zero field at a magnetic field of $10 \mathrm{G}$. As shown in Figure $1(\mathrm{~A})$, the transition is sharp with a $T_{c}$ (onset) $\simeq 42 \mathrm{~K}$, significantly higher than the maximum $T_{c}$ of $\sim 38 \mathrm{~K}$ for $\mathrm{La}_{1.85} \mathrm{Sr}_{0.15} \mathrm{CuO}_{4}$. The open symbols represent measurements taken on the same sample used in the neutron scattering experiments, both immediately after doping and 


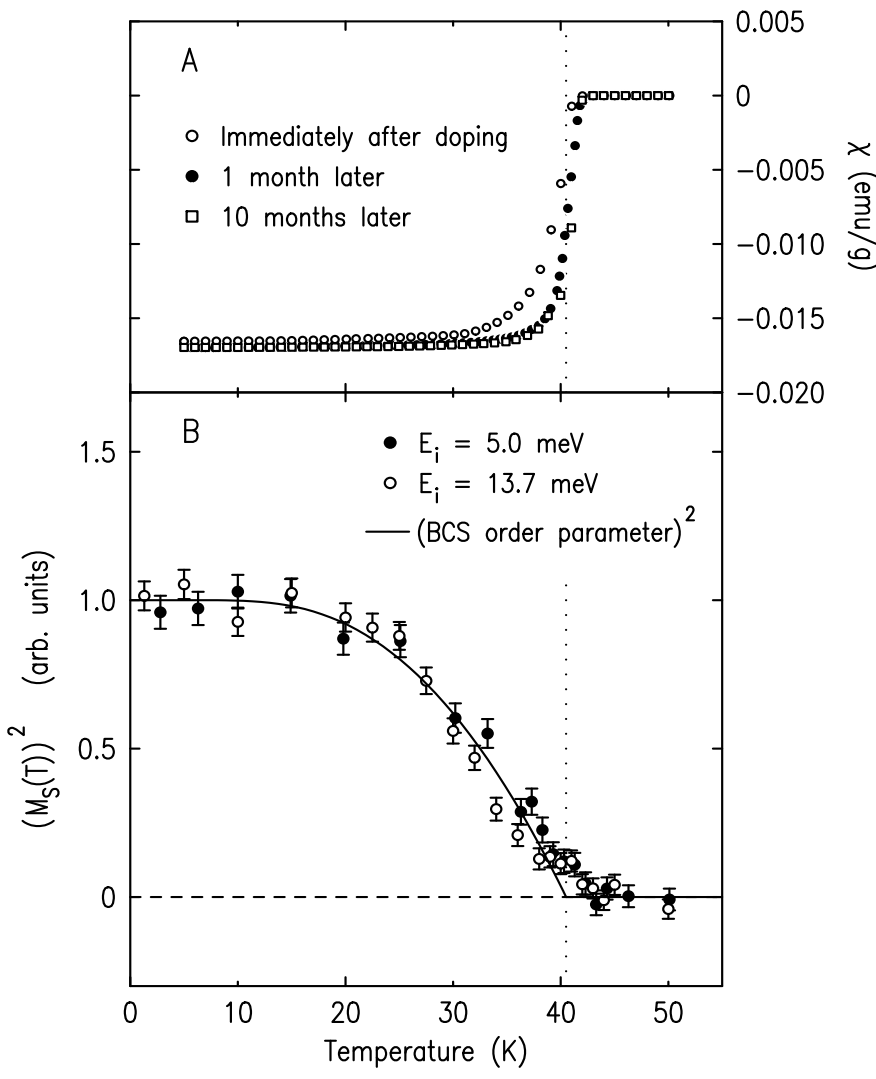

FIG. 1. A) Bulk magnetic susceptibility measured after several time intervals in an applied field of $10 \mathrm{G}$ after cooling in zero field. The open symbols represent data taken with the same crystal used in the neutron scattering experiments, while the closed symbols are taken on a small piece which was broken off of the original crystal immediately after doping. The vertical dotted line denotes the approximate midpoint temperature, $T_{c}$ (midpoint $)=40.5 \mathrm{~K}$, of the flux exclusion curve after the one month anneal. B) Temperature dependence of the peak intensity of the incommensurate elastic scattering, which is proportional to the square of the magnetic order parameter $M_{s}$. The measurements have been performed with two different neutron energies of $5.0 \mathrm{meV}$ and $13.7 \mathrm{meV}$, yielding energy resolutions of $0.075 \mathrm{meV}$ and $0.45 \mathrm{meV}$ (half-width at half-maximum), respectively with our particular choice of spectrometer collimations. The solid line denotes the square of the BCS order parameter, with $T_{m}=40.5 \mathrm{~K}=T_{c}$ (midpoint).

10 months later. The solid symbols represent data taken on a small piece broken off of the larger neutron scattering crystal. The two pieces had identical temperature dependences for their susceptibilities immediately after doping (not shown). To correct for their drastically different sample sizes, the susceptibilities of the two samples have been normalized to coincide at the lowest temperature. Previous studies of ceramic samples of electrochemically doped $\mathrm{La}_{2} \mathrm{CuO}_{4+y} 29$ have suggested that superconducting phases with $T_{c}>34 \mathrm{~K}$ may be unstable, exhibiting a significant diminution of $T_{c}$ after annealing at room temperature for several days. We find that for our single crystal sample, after a month-long anneal at room temperature, the superconducting $T_{c}$ does not decrease, but rather the transition sharpens to a slightly higher temperature. A 10 month anneal produces only a minimal additional effect. All of the neutron scattering data shown in this paper have been taken after the one month anneal. It is apparent, therefore, that the superconducting phase with a $42 \mathrm{~K}$ transition temperature onset in $\mathrm{La}_{2} \mathrm{CuO}_{4+y}$ is a stable phase. Attempts to measure the Meissner effect by cooling in a magnetic field yield a paramagnetic signal below $T_{c}$, which is not uncommon in studies of single frystal samples of $\mathrm{La}_{2} \mathrm{CuO}_{4}$-based superconductors. 13 26. In accordance with such previous studies, we believe that the zero-field-cooled measurements previde reliable evidence for bulk superconductivity.13, $17,30,31$

To estimate the oxygen doping level, thermogravitimetric analysis has been performed on similarly doped single crystals from the same boule; a separate sample was used so as not to de-oxygenate the sample used in the neutron scattering measurements. The oxygen weight loss yields a doping level of $y=0.12 \pm 0.005$, which is substantially higher than the oxygen doping range for macroscopic phase separation into superconducting and insulating phases. Reported titration measurement, 25,32 on powder samples of $\mathrm{La}_{2} \mathrm{CuO}_{4+y}$ imply that an oxygen level of $y \simeq 0.12$ corresponds to a hole concentration of $p \simeq 0.16$. Indeed, SQUID magnetometer measurements of the uniform magnetization at $T>T_{c}$ of our single crystal sample reveal identical scaling behavior as seen in $\mathrm{Sr}^{2+}$ doped samples3 34, with a $\mathrm{Sr}^{2+}$ doping level of $x \simeq 0.14 \pm 0.02$. Also, from ${ }^{63} \mathrm{Cu}$ nuclear quadrupole resonance (NQR) measurements, the NQR frequency as well as the quantitative behavior of $1 / T_{1}$ match those found in samples of $\mathrm{La}_{2-x} \mathrm{Sr}_{x} \mathrm{CuO}_{4}$ with $x \simeq 0.15 \pm 0.02 .35$ Therefore, the experimental evidence suggests that our crystal is a bulk superconductor with a distribution of holes similar in density and homogeneity to that of optimally doped, that is, $x \simeq 0.15, \mathrm{La}_{2-x} \mathrm{Sr}_{x} \mathrm{CuO}_{4}$ crystals. The data shown in Figure 1(B) will be described later in the discussion of the spin density wave peaks.

\section{STAGING BEHAVIOR}

Our oxygen doped crystal is orthorhombic at low temperatures. Undoped $\mathrm{La}_{2} \mathrm{CuO}_{4}$ is also orthorhombic at low temperatures with the Bmab space group symmetry, which differs from tetragonal symmetry because of the tilt pattern of the $\mathrm{CuO}_{6}$ octahedra. One $\mathrm{CuO}_{6}$ octahedron is depicted in the unit cell diagram in Figure 2(A); the tilt pattern causes a slight buckling of the $\mathrm{CuO}_{2}$ plane as shown in Figure 2(B), yielding the orthorhombic symmetry. In $\mathrm{La}_{2} \mathrm{CuO}_{4+y}$, the interstitial oxygens modify the structure along the $c$-direction by periodically introducing planes across which the tilt pattern reverses. The segregation of excess oxygen into periodic planes, known as staging, has been observed and explained previously 
by Wells et al. 3.36 for single crystals of $\mathrm{La}_{2} \mathrm{CuO}_{4+y}$. Stage $n$ refers to a structure in which the tilt reversal occurs periodically every $n \mathrm{CuO}_{2}$ layers. Therefore, increasing the excess oxygen content leads to structural phases with successively smaller staging numbers $n$. At low oxygen concentrations $(0.012 \lesssim \delta \lesssim 0.055)$, for which phase separation into oxygen-rich and oxygen-poor domains occurs, the oxygen-rich phase is stage- 6 with a superconducting $T_{c}$ of 31-32 K⿺辶27. 88 while the oxygen-poor phase is an insulating antiferromagnet. For higher oxygen concentrations $(\delta>0.055)$, the crystal remains entirely oxygen rich and stages 4,3 , and 2 are seen. 36 Throughout this paper, we use reciprocal lattice units (r.l.u.) for the Bmab low temperature orthorhombic crystal structure, for which the axes are depicted in Figure 2. The reciprocal space $(H, K, L)$ components are given in units of $(2 \pi / a, 2 \pi / b, 2 \pi / c)$, where, at low temperature, the inplane lattice constants are $a=5.333 \AA$ and $b=5.401 \AA$, and the out-of-plane lattice constant is $c=13.18 \AA$.

We find, via neutron diffraction, that our $\mathrm{La}_{2} \mathrm{CuO}_{4+y}$ crystal predominately possesses the tilt structure corresponding to stage-4; however smaller peaks corresponding to stage- 2 and other staged phases are seen as well. In Figure 3, we show a scan through the staging superlattice peaks which straddle the (014) Bmab peak position along the $L$ direction. For each stage $n$ phase present, corresponding staging peaks occur in pairs, split by $\pm 1 / n$ from the Bmab position. The line through the data represents the results of fits to pairs of staging peaks using Gaussian lineshapes, convolved with the resolution. The data indicate that the components with stage- 4 , fitted with $n=3.97(3)$, and stage-2, fitted with $n=2.01(1)$, account for $80 \%$ of the diffraction intensity in this scan. Additionally, the integrated intensity of the stage- 4 peaks is 2.0 times larger than that of the stage-2 peaks. We find that the stage- 2 peaks are resolution limited in the $L$ direction, whereas the stage- 4 peaks have a non-zero width indicating a domain size of $\sim 240 \AA$ along the $L$ direction. Both the stage- 4 and stage- 2 peaks are resolution limited along the in-plane $K$ direction. This comparison of the integrated intensities of the staging peaks gives only an approximate measure of the phase fractions present in the crystal. To be more accurate, one needs to account for the structure factor of the staged phases, which depends significantly on the amplitude of the tilting of the $\mathrm{CuO}_{6}$ octahedra. However, to a first approximation, assuming a uniform amplitude of the octahedral tilt throughout the crystal, the magnitudes of the structure factors for the staged phases are nearly equal. Minority phases with stage-6, stage-3, and undoped Bmab structures account for the rest of the diffraction peaks. This confirms that integer values for staging numbers appear to give the most stable phases, and that stage- 5 , which has yet to be observed in high quality single crystals, is probably not stable.

Doping with mobile oxygen interstitials has the advantage of yielding a lower degree of dopant
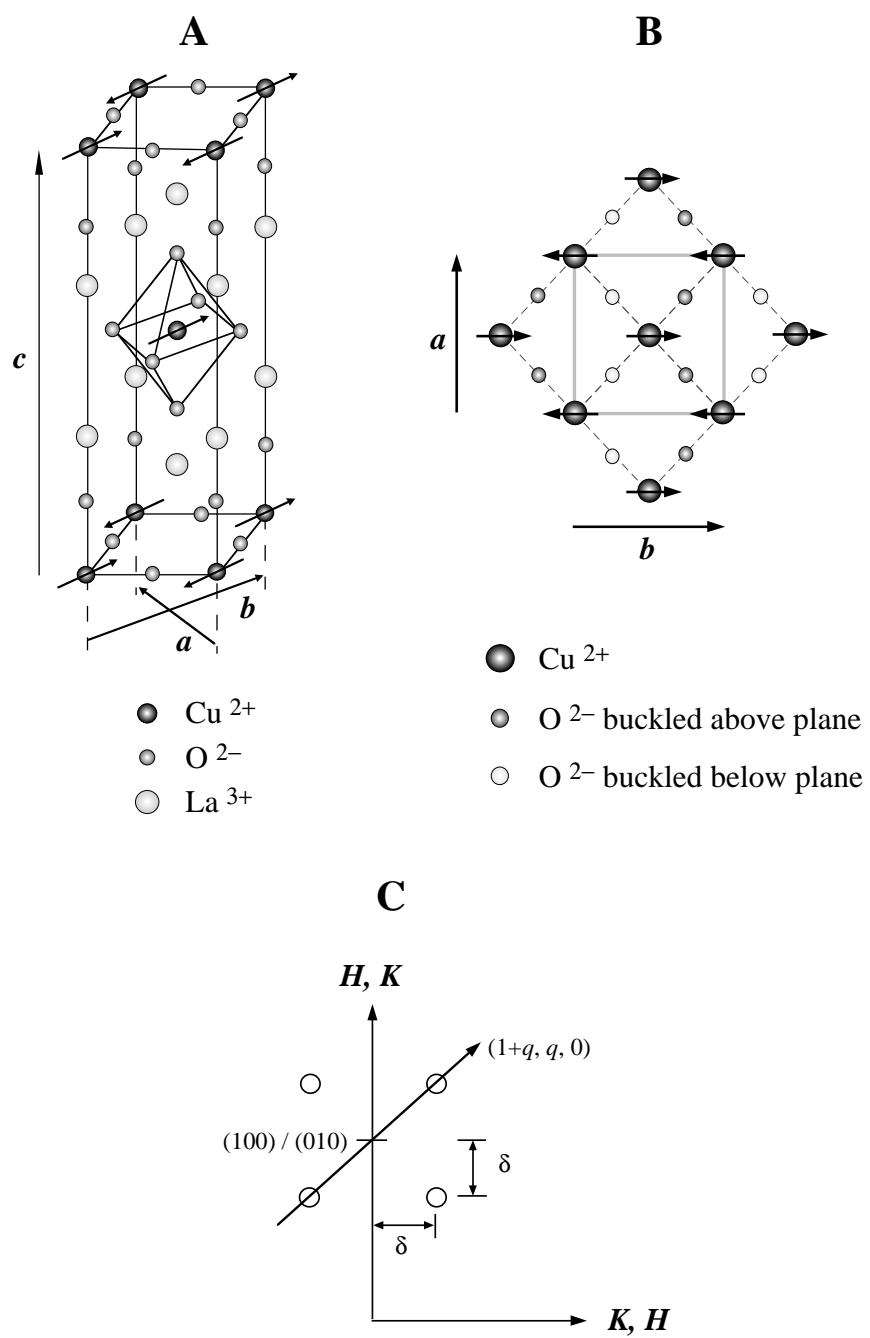

FIG. 2. A) Unit cell of $\mathrm{La}_{2} \mathrm{CuO}_{4}$ depicting the orthorhombic axes. B) Diagram of the copper-oxygen plane, highlighting the $2 \mathrm{D}$ orthorhombic unit cell. C) Reciprocal space map of the positions of the inelastic incommensurate magnetic peaks in superconducting $\mathrm{La}_{2-x} \mathrm{Sr}_{x} \mathrm{CuO}_{4+y}$. The trajectory of the inelastic scattering scans in Figure $4 \mathrm{~A}$ is denoted by the line $(1+q, q, 0)$.

disorder. In fact, we have recently succeeded in observing scattering directly from the ordered distribution of interstitial oxygen, at reciprocal lattice positions distinct from the staging superlattice peaks 37 The annealed character of the oxygen disorder is revealed in measurements linking a reduction of the superconducting $T_{c}$ to the loss of the ordered oxygen lattice. 37 A possible disadvantage of having mobile oxygens is that it may be easier for the crystal to become macroscopically inhomogenous by phase separating into regions with different hole concentrations. The presence of multiple staged phases in our crystal implies that the oxygen concentration may vary between the macroscopic domains with different staging numbers. It has been shown that the oxygen 


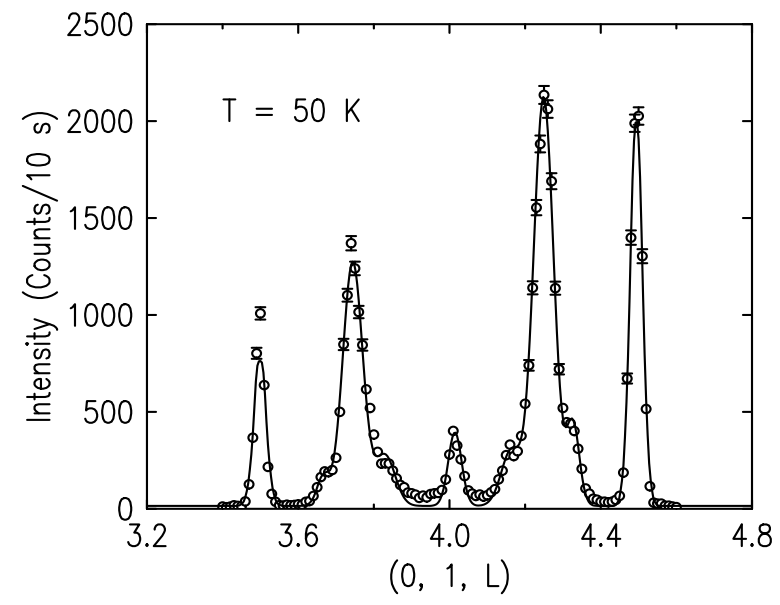

FIG. 3. Scan along the $L$ direction through the staging superlattice positions at $T=50 \mathrm{~K}$ measured with $5 \mathrm{meV}$ neutrons and horizontal collimations of $30^{\prime}-40^{\prime}-S-20^{\prime}-B$, where $S$ denotes the sample and $B$ is a blank collimator. The solid line denotes the results of a fit to pairs of staging peaks centered about the (014) position in addition to a small Bmab peak at (014), convolved with the instrumental resolution.

concentration sharply increases between the stage- 6 phase, with $y=0.055$, and the stage- 4 phase, with $y \simeq 0.11 .27$ However, it is not known how the interstitial oxygen concentration varies between the stage- 4,3 , and 2 phases, which account for most of the volume of our crystal. Other electrochemical studies on ceramics and single crystals of $\mathrm{La}_{2} \mathrm{CuO}_{4+y}$ report that the oxygen doping level produced by this method saturates at a maximum value of $y \sim 0.12 .25 .26$. 32 Therefore, it appears likely that the oxygen concentration does not vary substantially between the stage- 4,3 , and 2 phases. This is eqnsistent with the previous measurements of Wells et al. 3 which show that the $c$-axis lattice constant is the same for a crystal with a single stage- 4 phase and one with simultaneous stage- 4,3 , and 2 phases. High resolution measurements of the $c$-axis lattice constant for our crystal give no indication that the sample has more than a single oxygen doped phase. Thus, we conclude that our sample is both highly oxygen doped and completely oxygen-rich throughout its bulk. Also, we reiterate that measurements which depend on hole density indicate that our $\mathrm{La}_{2} \mathrm{CuO}_{4+y}$ sample behaves similarly to $\mathrm{La}_{2-x} \mathrm{Sr}_{x} \mathrm{CuO}_{4}$ with $x \sim 0.15$. Therefore, the doped holes in this oxygen-rich sample seem to be as uniformly distributed throughout the bulk as those in Sr-doped $\mathrm{La}_{2-x} \mathrm{Sr}_{x} \mathrm{CuO}_{4}$, and have an effective hole concentration of $n_{h} \simeq 0.15 \pm 0.02$. This is consistent with our observation of a single, sharp superconducting transition at $T_{c}$ (onset) $=42 \mathrm{~K}$. In addition, as will be discussed next, our neutron scattering meausurements of the magnetism are consistent with scattering from a sample with a single hole concentration.

We should note that we have recently developed a technique for electrochemically doping $\mathrm{La}_{2} \mathrm{CuO}_{4+y}$ which ap- pears to produce pure stage- 4 material. 77 However, at the present time this uniformity in the staging number is offset by a slight decrease in $T_{c}$ and increased oxygen disorder. We plan neutron scattering experiments on these pure stage- 4 systems in the near future.

\section{INELASTIC MAGNETIC NEUTRON SCATTERING}

In previous ipelastic neutron scattering work on stage$6 \mathrm{La}_{2} \mathrm{CuO}_{4+y} \mathrm{~B}$, incommensurate magnetic excitations have been confirmed to be universal in the $\mathrm{La}_{2} \mathrm{CuO}_{4}{ }^{-}$ based superconductors. The magnitude and direction of the incommensurate wavevector follows the same pattern as that for superconducting $\mathrm{Sr}^{2+}$ doped $\mathrm{La}_{2} \mathrm{CuO}_{4}$. particular, the behavior of $\chi^{\prime \prime}(\mathbf{Q}, \omega)$ for $\mathrm{La}_{2} \mathrm{CuO}_{4+y}$ with $T_{c}=31 \mathrm{~K}$ is essentially identical to that for underdoped $\mathrm{La}_{2-x} \mathrm{Sr}_{x} \mathrm{CuO}_{4}$ with similar $T_{c}$, in which a partial suppression of the intensity of the spin excitations is seen as the temperature is reduced below the superconducting $T_{c}$. This contrasts with the behavior in optimally doped $\mathrm{La}_{2-x} \mathrm{Sr}_{x} \mathrm{CuO}_{4}$ with $x=0.15$, in which a complete gap is observed in the magnetic excitation spectrum in the low temperature superconducting state for energies $\leq 3.5 \mathrm{meV} .39$ This difference is probably related to the apparent absence of spert-range or long-range SDW order in $\mathrm{La}_{1.85} \mathrm{Sr}_{0.15} \mathrm{CuO}_{4} 20$, whereas in the underdoped samples SDW order occurs in the superconducting state.

We have performed inelastic neutron scattering measurements on our $\mathrm{La}_{2} \mathrm{CuO}_{4+y}$ sample probing the magnetic excitations with an energy transfer of $2 \mathrm{meV}$ both above and below the superconducting transition. The low-energy spin fluctuations in $\mathrm{La}_{2} \mathrm{CuO}_{4}$-based superconductors consist of four peaks equally displaced from the antiferromagnetic zone center by an incommensurate wavevector $\delta$ along each of the four $\mathrm{Cu}-\mathrm{O}-\mathrm{Cu}$ directions. This arrangement is depicted in Figure $2(\mathrm{C})$, where the (100) and (010) orthorhombic positions would be equivalent to each other if the crystal were tetragonal, and both correspond to the $\left(\frac{1}{2} \frac{1}{2} 0\right)$ position in tetragonal notation. Scans through two of the incommensurate peak positions have been taken along the trajectory $(1+q, q, 0)$ as shown in Figure 2(C). Representative data collected at $T=2 \mathrm{~K}$ and $T=44 \mathrm{~K}$ are plotted in Figure $4(\mathrm{~A})$. The solid lines are the results of fits to 2D Lorentzians convolved with the instrumental resolution; the Lorentzian width is found to be less than $0.008 \AA^{-1}$ half-width at halfmaximum (HWHM) and is independent of temperature within the errors. Such a narrow width is also seen for $\mathrm{La}_{2-x} \mathrm{Sr}_{x} \mathrm{CuO}_{4}$ crystals with a doping level close to 1/8.2 (Note that there is a weak contaminant peak at $q \simeq 0.15$.) The incommensurability is found to be $\delta \simeq 0.121(2)$. The temperature dependence of $\chi^{\prime \prime}\left(\mathbf{Q}_{\delta}, \omega\right)$ which is presented in Figure 4B, is strikingly different from that for the optimal $\mathrm{Sr}^{2+}$ doped material $\mathrm{La}_{1.85} \mathrm{Sr}_{0.15} \mathrm{CuO}_{4}$ 

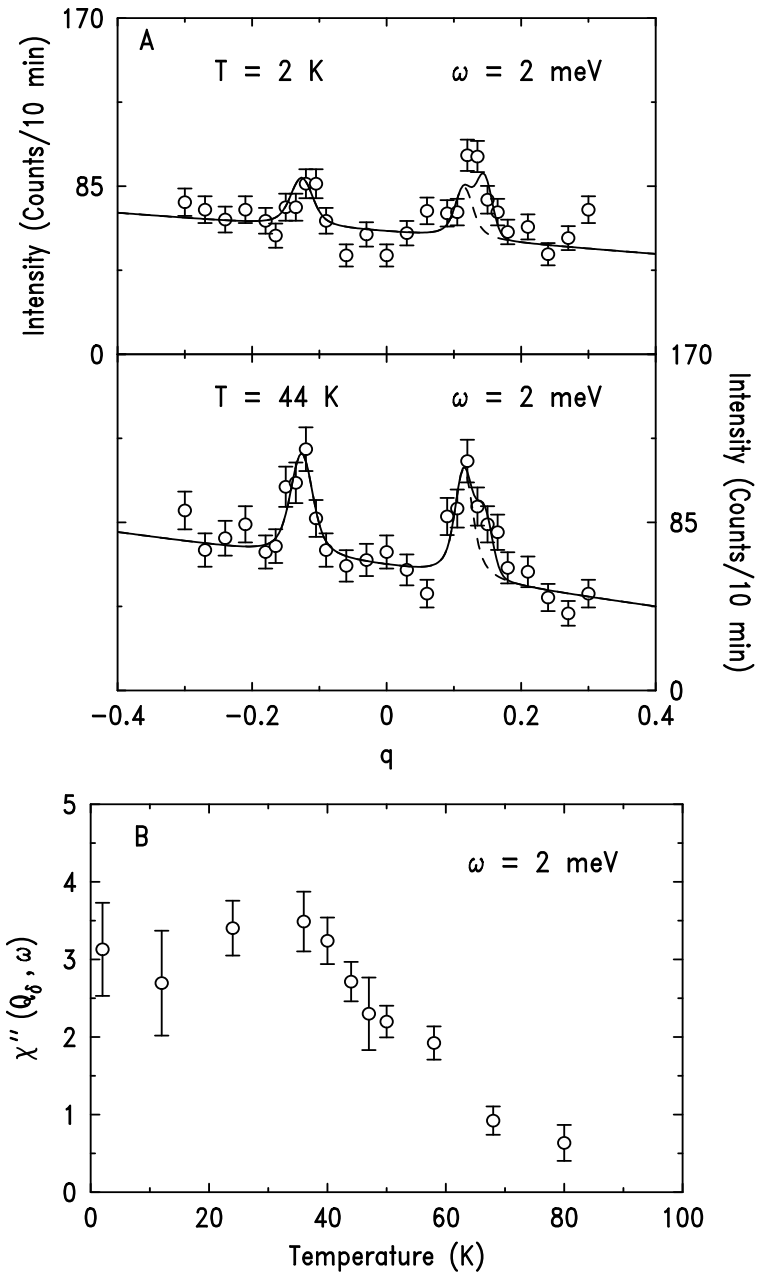

FIG. 4. A) Inelastic scans through the incommensurate positions along the trajectory given in Figure $2 \mathrm{C}$ at $T=2 \mathrm{~K}$ and $T=44 \mathrm{~K}$ for an energy transfer of $\omega=2 \mathrm{meV}$, measured with $14.7 \mathrm{meV}$ neutrons and horizontal collimations of $B-60^{\prime}-S-60^{\prime}-B$. The solid lines denote the results of fits to the cross section described by four incommensurate peaks, together with a sloping background and a weak, temperature-independent, contaminant peak on the shoulder of the high- $q$ peak. The dashed line at the high- $q$ position denotes the magnetic signal. B) The imaginary part of the susceptibility, $\chi^{\prime \prime}\left(\mathbf{Q}_{\delta}, \omega\right)$, extracted from the intensity of the scattering at the incommensurate positions via Eq. 4.

with $T_{c}=37.5 \mathrm{~K} 39$ We see that, above the superconducting transition temperature, the measured susceptibility increases continuously as the temperature decreases. However, below $T_{c}$ the dynamic susceptibility levels off and approaches a non-zero constant value as $T \rightarrow 0$, whereas for $\mathrm{La}_{1.85} \mathrm{Sr}_{0.15} \mathrm{CuO}_{4}$ the intensity diminishes to zero at low temperature. This is a clear difference between oxygen doping and $\mathrm{Sr}^{2+}$ doping; even though both $T_{c}$ 's appear to be optimal, the low energy magnetic excitations behave differently in the superconducting state. As we shall discuss below, the non-zero intensity at low temperatures in the $\mathrm{La}_{2} \mathrm{CuO}_{4+y}$ sample arises from scattering from spin wave excitations associated with the SDW. A few remarks are appropriate at this point. First, the $2 \mathrm{meV}$ inelastic peaks are sharp in reciprocal space, and only a single set is seen. Previous systematic studies in $\mathrm{La}_{2-x} \mathrm{Sr}_{x} \mathrm{CuO}_{4}$ show that the incommensurability of the inelastic scattering depends sensitively on the hole doping level for $x \lesssim 0.125$ and saturates around $1 / 8$ for doping levels $x \gtrsim 0.125$. Therefore, since the observed value of the inelastic incommensurability $\delta$ in our sample is near $1 / 8$, this implies that the hole doping level is also near $1 / 8$ or possibly higher, which would be consistent within the errors with our deduction above that $n_{h}=0.15 \pm 0.02$. Additionally, since the width we measure is as small as that of the narrowest peaks observed in $\mathrm{La}_{2-x} \mathrm{Sr}_{x} \mathrm{CuO}_{4}$, the incommensurate inelastic scattering is strong evidence for a homogenous distribution of holes in our sample. Even if there were a distribution of hole concentrations in our crystal, the observed magnetic scattering would be attributable to at least one of these concentrations, and all such high oxygen doping concentrations are known to be superconducting 24,25 Therefore, we conclude that these sharp low-energy magnetic fluctuations and the superconductivity coexist in the same doped phase.

\section{SPIN DENSITY WAVE ORDERING}

The most important finding of this study is that we observe sharp elastic magnetic scattering at low temperatures. The observed elastic peak positions are shown in Figure 5. Due to the twinning of this orthorhombic crystal, which is tetragonal at high temperatures, the neutrons simultaneously scatter from four twin domains. Two of the twin domains have different in-plane lattice constants along the same direction; thus we simultaneously see both the (020) and (200) nuclear Bragg peaks in a single longitudinal scan. In addition, each of these domains has another twin counterpart which is rotated about the $c$-axis by a small angle $\left(\sim 0.7^{\circ}\right)$ which is proportional to the size of the orthorhombic distortion. Since the twinning can be precisely characterized via scattering from the nuclear Bragg peaks, there is little ambiguity in interpreting the magnetic scattering.

Almost all previous neutron scattering measurements of the inelastic incommensurate peaks have used a relatively wide resolution to optimize the signal to background ratio, since the intrinsic signal is found to be extended in energy. In our measurements of the elastic scattering, we find that the signal is narrow in both energy and wavevector. Therefore, we employ a relatively narrow resolution to obtain a precise measurement of the peak positions and widths in reciprocal space. Taking a narrow mesh of scans in the $(H K 0)$ scattering plane, we find two twin-related sets of four incommensurate peaks which share a common center at the 


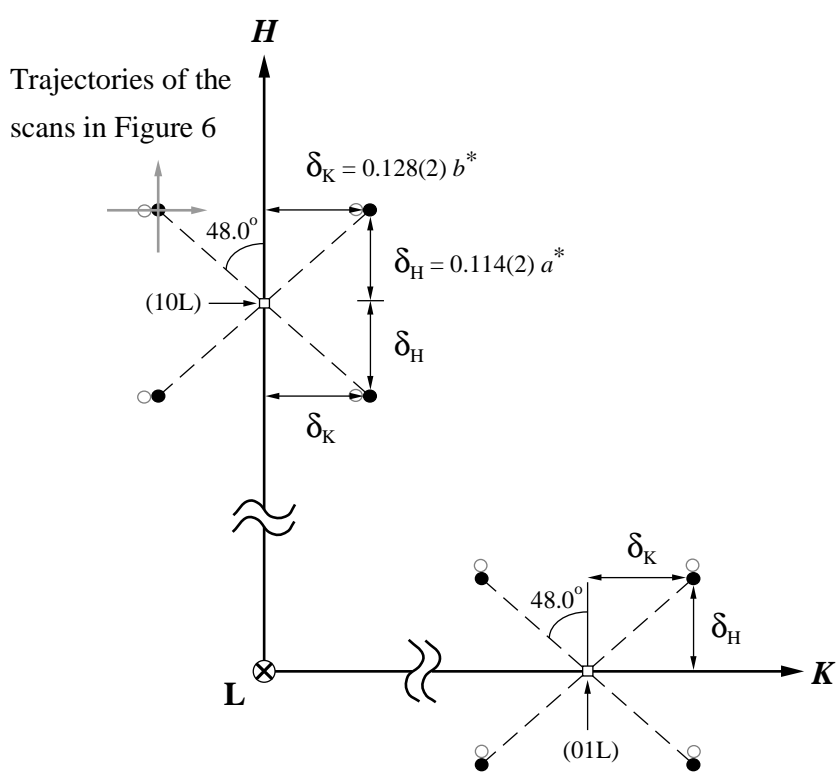

FIG. 5. Positions of the elastic incommensurate spin density wave peaks (closed circles) in a single structural twin domain, deduced from our high resolution measurements. $H$ and $K$ are in orthorhombic units. The open circles represent SDW peaks originating from another twin domain, which we measure simultaneously in our scans. The trajectories for the scans in Figures $6(\mathrm{~A})$ and $6(\mathrm{~B})$ are denoted by the arrows through the position $\left(1+\delta_{H},-\delta_{K}, 0\right)$.

(100) position, and not the (010) position as shown in the upper portion of Figure 5. Therefore, each set of four peaks almost certainly originate from a single structural twin domain. In fact, we also find incommensurate peaks about the $(01 L)$ position (as shown in the lower portion of Figure 5), but at odd-integer $L$ values, which we discuss further below. The intensities of the four peaks are observed to be identical within the errors. Surprisingly, we see that the incommensurate wavevector is not precisely aligned with the $\mathrm{Cu}-\mathrm{O}-\mathrm{Cu}$ tetragonal direction, but has different incommensurabilities along the orthorhombic $H$ and $K$ directions: $\delta_{H} \simeq 0.114(2) a^{*}$ and $\delta_{K} \simeq 0.128(2) b^{*}$. This corresponds to a rotation of the incommensurate wavevector by $3.3^{\circ}$ with respect to the $\mathrm{Cu}-\mathrm{O}-\mathrm{Cu}$ direction.

We show in Figures 6(A) and 6(B) elastic scans through the incommensurate elastic peak depicted by the trajectories illustrated by the arrows in the upper left corner of Figure 5. The measurements have been performed with $5 \mathrm{meV}$ neutrons and the horizontal collimations are $30^{\prime}-20^{\prime}-S-20^{\prime}-B$, where $S$ denotes the sample and $B$ is a blank collimator. The observed peaks are extremely sharp and indeed are close to being resolution limited. The solid lines in the figure are 2D Gaussian line-shapes (with a delta function in energy) convolved with the instrumental resolution. The double peak structure in Figure 6(B) results from structural
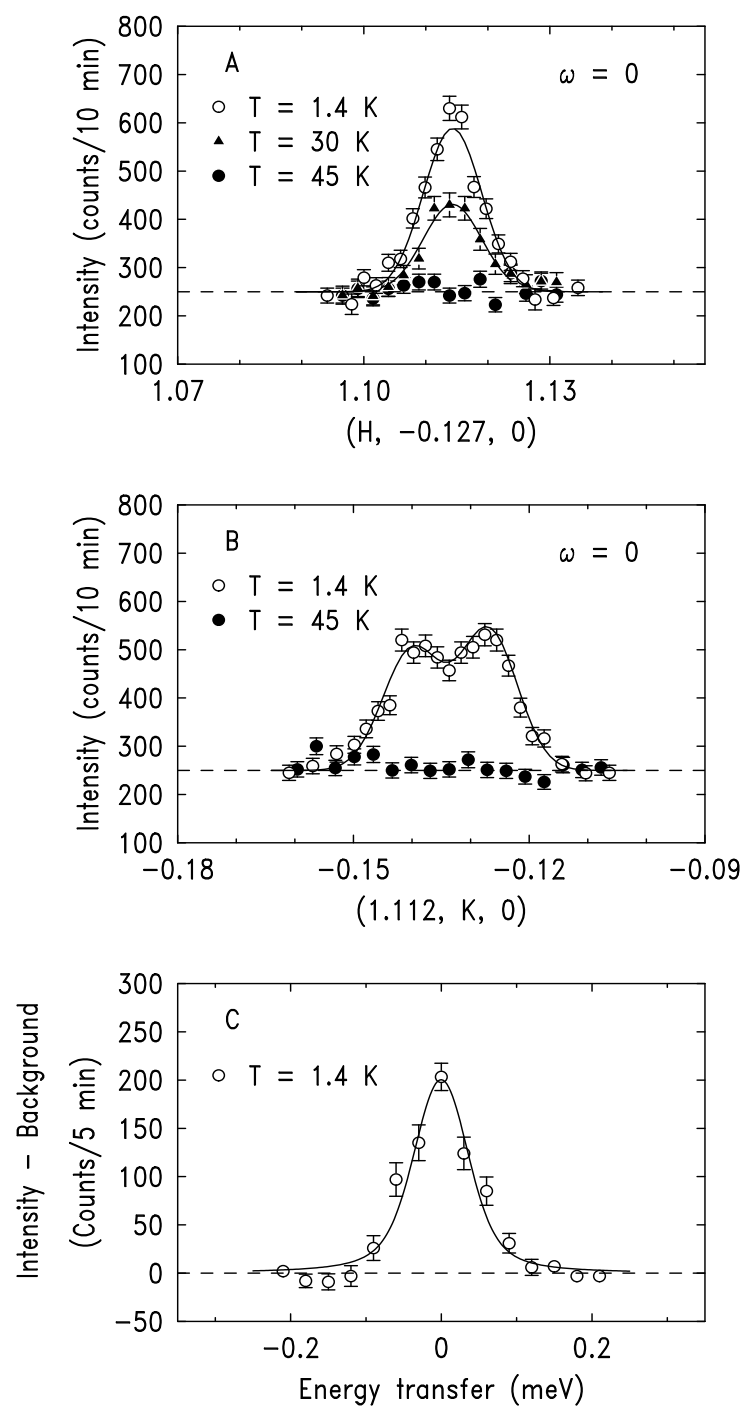

FIG. 6. Elastic scans through the incommensurate peak position. A) Scans along the orthorhombic $H$ direction, as depicted in Figure 5, for various temperatures. B) Scans along the orthorhombic $K$ direction, as depicted in Figure 5 . C) Scan of the energy transfer at a fixed incommensurate Q-position. The solid lines denote the results of fits to the cross section convolved with the resolution as described in the text.

twinning. Fits to either Gaussian or Lorentzian intrinsic line-shapes can adequately describe the data to within the errors and both indicate that the in-plane static magnetic order is isotropically correlated over distances greater than $400 \AA$. This lower bound on the extent of the long-range in-plane spin correlations is limited by the uncertainty in the instrumental resolution, which we could quantify to within $10 \%$ for the transverse direction. Figure $6(\mathrm{C})$ displays data in which the energy transfer is scanned while the reciprocal space position is fixed at the peak position of the elastic signal. The observed energy width of the peak is also very narrow. The solid line is 

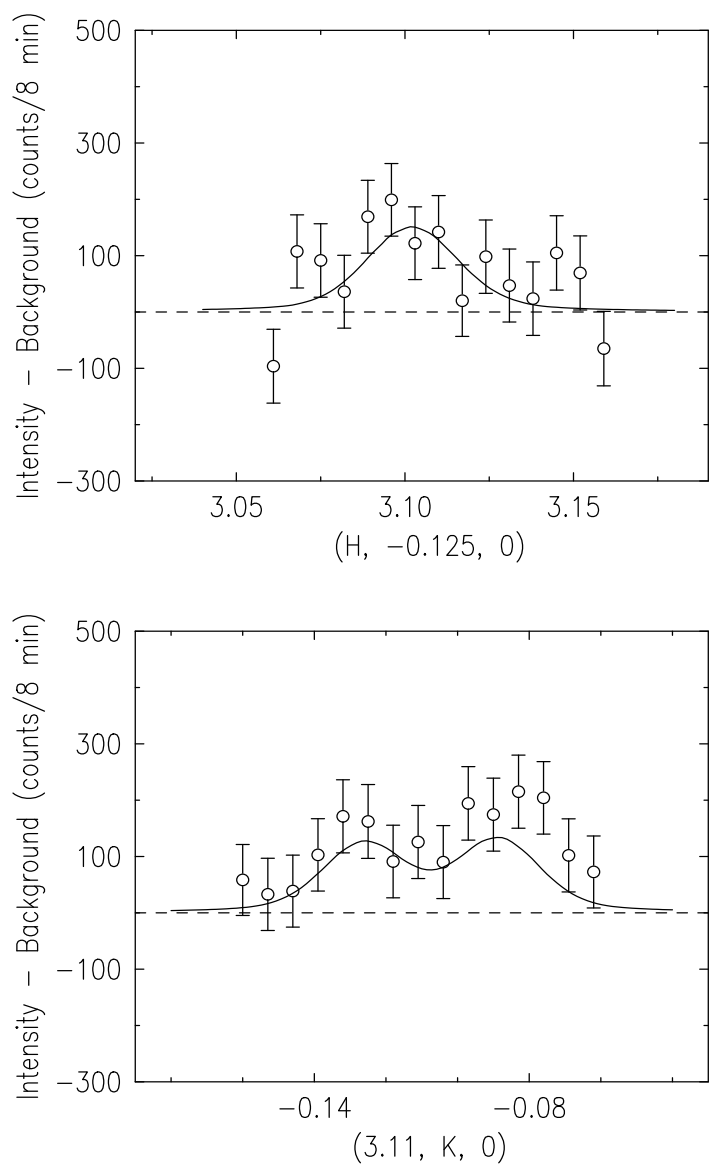

FIG. 7. Elastic scans through the incommensurate peaks positions near the (300) antiferromagnetic Brillioun zone center position, measured with $13.7 \mathrm{meV}$ neutrons and horizontal collimations of $30^{\prime}-30^{\prime}-S-30^{\prime}-B$. The background signal at $T=50 \mathrm{~K}$ has been subtracted from the data taken at $T=2 \mathrm{~K}$. The solid lines denote the results a fit to the cross section convolved with the resolution as described in the text. The reduction in intensity here relative to that measured around the (100) position is consistent with the $\mathrm{Cu}^{2+}$ magnetic form factor in $\mathrm{La}_{2} \mathrm{CuO}_{4}$.

a Lorentzian lineshape in energy transfer convolved with the instrumental resolution, assuming the above narrow $q$-widths. The results of the fit indicate that the intrinsic energy width of the peak is less than $\sim 0.025 \mathrm{meV}$ HWHM at $T=1.4 \mathrm{~K}$; the instrumental energy resolution is $0.075 \mathrm{meV}$ HWHM. We also observe incommensurate peaks around the (300) position, albeit with much weaker intensity as shown in Figure 7. After taking into account the instrumental resolution including the Lorentz factor and the crystal mosaic, we find that the intrinsic signal around (300) is smaller by a factor of approximately 2.5 than that around (100). This is consistent with the decrease in signal that is expected from the $\mathrm{Cu}^{2+}$ magnetic form factor in undoped $\mathrm{La}_{2} \mathrm{CuO}_{4} 40$. By contrast, for nuclear scattering the intrinsic signal should increase by $Q^{2} \sim 9$ between (100) and (300). Thus, we conclude that the observed incommensurate elastic scattering is magnetic in origin.

We next address the question of how the static spin arrangement is correlated between successive copper oxide planes. For this experiment, the sample is remounted on the spectrometer so that the $c$-axis and an orthorhombic in-plane axis are both in the scattering plane. In this way, reciprocal space positions corresponding to $(H 0 L)$ may be reached, and, as a result of the twinning, $(0 K L)$ positions are simultaneously measured. In order to scan through the incommensurate position, we must tilt the sample by $8^{\circ}$ to bring one of the incommensurate peaks into the scattering plane. From measuring the intensity of a "(10L)-centered" incommensurate peak at $L=0$, we find that the intensity is a factor of 4 times smaller than that measured in the $(H K 0)$ configuration. This is not surprising, since the neutron spectrometer has a broad vertical resolution which integrates the signal over a broad width of reciprocal space perpendicular to the scattering plane; hence the reduction in the detected intensity in this configuration suggests that the intrinsic signal is composed of diffuse peaks along the $L$ direction. Indeed, in pure $\mathrm{La}_{2} \mathrm{CuO}_{4}$ the magnetic exchange between nearest neighbor planes is four orders of magnitude smaller than the exchange within the plane, so the spins exhibit observable correlations along this outof-plane direction only in the Néel phase.

By fixing the in-plane component of the $\mathbf{Q}$-vector to lie on the incommensurate position $\left(1-\delta_{H}, \delta_{K}, L\right)$, we perform scans along the $L$ direction through the peak. Scans have been made at $T=2 \mathrm{~K}$ to measure the elastic magnetic intensity and have been repeated at $T=50 \mathrm{~K}$ to measure the background. The background is not strictly flat, so we performed $H$-scans at several fixed $L$-positions and verified that the peaks are resolution limited for the in-plane direction and that the appearance of this resolution limited SDW peak is the only change in the scattering between $50 \mathrm{~K}$ and $2 \mathrm{~K}$. In Figure 8(A), we show the $L$-dependence of this scattering. There is a clear modulation of the signal with maxima at even-integer $L$ values. The peaks are broad, suggesting short-range spin correlations between the copper-oxide planes. We have also performed measurements with the alignment such that the measured incommensurate position was centered about the $(01 L)$ position; that is, we fix the $\mathbf{Q}$-vector to lie on the $\left(\delta_{H}, 1-\delta_{K}, L\right)$ incommensurate position. The $L$-dependence of the magnetic intensity of this " $(01 L)$ centered" domain is shown in Figure 8(B).

The intensity modulation of both the $(10 L)$ - and $(01 L)$-centered scattering is reminiscent of the ordered spin structure of the undoped insulating parent compound $\mathrm{La}_{2} \mathrm{CuO}_{4}$, which has the spin direction along (010) and the antiferromagnetic propagation vector along (100). 11 Specifically, since $\mathrm{La}_{2} \mathrm{CuO}_{4}$ has an orthorhombic structure, the magnetic exchange between planes is not perfectly frustrated. Thus, a particular 3D stacking arrangement of the spins on nearest neighbor planes is observed, in which the inter-plane nearest neighbor 

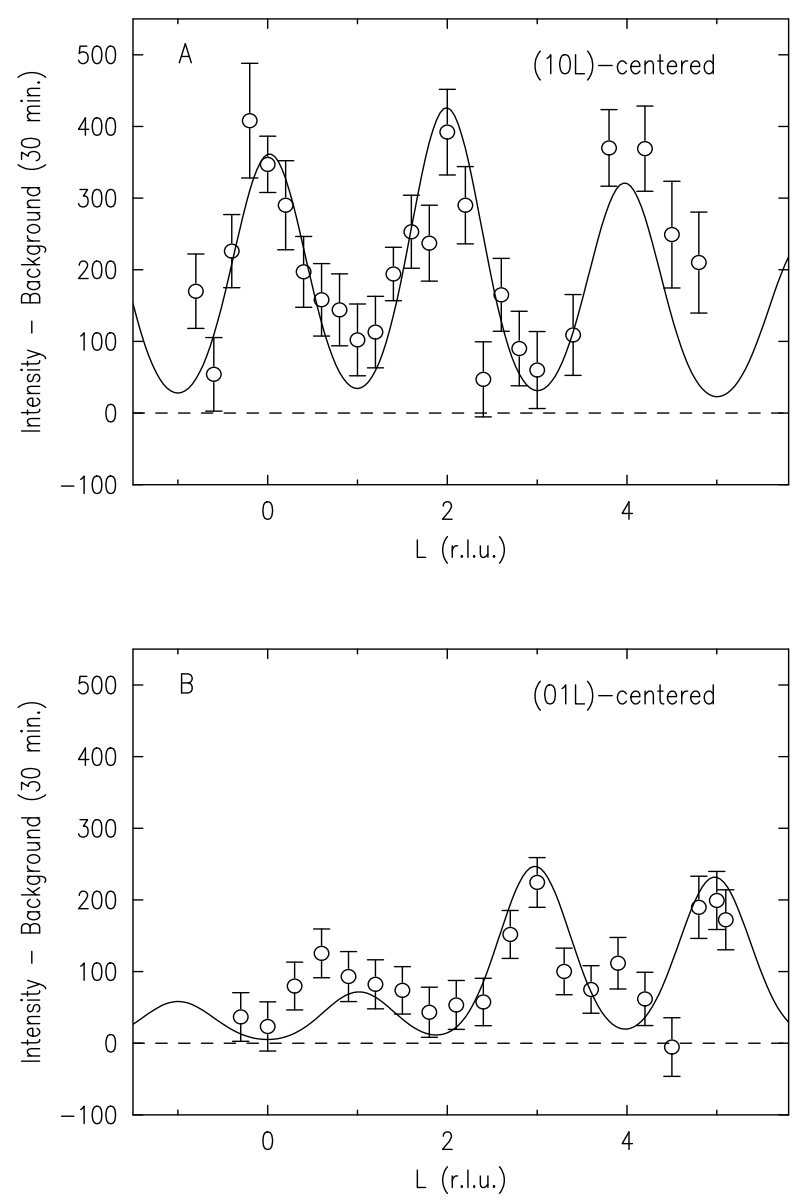

FIG. 8. Elastic scans along the $L$ direction perpendicular to the $\mathrm{CuO}_{2}$ planes, measured with $5 \mathrm{meV}$ neutrons and horizontal collimations of $30^{\prime}-40^{\prime}-S-20^{\prime}-B$. A) Scan through the incommensurate position centered about $(10 L)$, scanning along $\left(1-\delta_{H}, \delta_{K}, L\right)$. B) Scan through the incommensurate position centered about $(01 L)$, scanning along $\left(\delta_{H}, 1-\delta_{K}, L\right)$. The solid lines denote the results of a fit assuming a model for the stacking arrangement and spin direction identical to that in pure $\mathrm{La}_{2} \mathrm{CuO}_{4}$, as described in the text.

spins are aligned anti-parallel, yielding magnetic Bragg peaks at $(H 0 L)$, with $H$ odd and $L$ even, and $(0 K L)$, with $K$ odd and $L$ odd. In addition, the work of Thio et al. 22 has shown that in pure $\mathrm{La}_{2} \mathrm{CuO}_{4}$ the DzyaloshinskiMoriya anti-symmetric exchange term in the spin Hamiltonian in the orthorhombic phase favors spin alignment along the $K$ direction, which is also observed here. The solid lines in both panels of Figure 8 represent the results of fits to Gaussian lineshapes in the $L$ direction (along with Bragg peaks for the in-plane cross section as discussed above) convolved with the instrumental resolution assuming a model for the stacking arrangement and spin direction identical to that in pure $\mathrm{La}_{2} \mathrm{CuO}_{4}$. Here, the only free parameters are the width of the peaks in $L$ and a single overall intensity scale factor. The agree- ment is clearly satisfactory, and the fitted width indicates that, within a finite-size domain model, the spins are correlated over $\sim 13 \AA$ in the $L$ direction, or across 2-3 $\mathrm{CuO}_{2}$ planes. As can be seen from Eq. 3, if the spins order collinearly, the neutron cross section depends on the angle $\theta$ between the $\mathbf{Q}$ scattering vector and the ordered spin direction by a factor which can be written as $\sin ^{2}(\theta)$. The relative intensities of all of the peaks in Figure 8, especially the growth in the peak intensity for the $(01 L)$-centered scattering for increasing $L$ shown in Figure 8(B), are consistent with this geometrical factor if the copper spin direction is fixed along the (010) direction. We have explicitly verified via $K$-scans that the $(01 L)$-centered scattering at $L=3$ is significantly greater than that at $L=1$ and is resolution limited for this in-plane direction. Here, the magnetic form factor is assumed not to vary significantly over the range of $L$ values scanned here, similar to the situation in pure $\mathrm{La}_{2} \mathrm{CuO}_{4} 40.43$ However, we do account for instrumental resolution effects which weakly modify the intensity over the course of the scan. We conclude that the stacking arrangement of the magnetically ordered planes in our $\mathrm{La}_{2} \mathrm{CuO}_{4+y}$ sample is the same as that of the undoped Mott insulator $\mathrm{La}_{2} \mathrm{CuO}_{4}$, even though the material is superconducting and the magnetic scattering is incommensurate. This is direct and compelling evidence that the magnetism of the doped superconductors is related to the magnetism in the undoped insulators in a very specific way.

We show in Figure 1(B) the peak intensity of the elastic signal as a function of temperature measured using both $5 \mathrm{meV}$ and $13.7 \mathrm{meV}$ neutrons. The fact that one obtains identical results for the temperature dependences of the intensities with these two different neutron energies and, concomitantly, energy resolutions implies that the scattering is truly elastic. We note that since the peak widths do not vary with temperature to within the errors, the peak intensity is proportional to the integrated intensity. The intensity of the elastic scattering turns on at approximately the same temperature as the onset of superconductivity. With decreasing temperature, the intensity increases at first approximately linearly and then crosses over to a saturation level at the lowest temperatures. Noting that the intensity of the magnetic scattering is proportional to the square of the magnetic order parameter, we have plotted the square of the BardeenCooper-Schrieffer (BCS) weak coupling order parameter curve together with the data using a $T_{m}$ of $40.5 \mathrm{~K}$ equal to $T_{c}$ (midpoint) $\simeq 40.5 \mathrm{~K}$. The good agreement demonstrates, at the minimum, that the magnetism exhibits mean field behavior. This is very surprising given the two dimensionality of the ordered magnetism.

The size of the average ordered moment at base temperature has been estimated several ways. We compare the magnetic signal to that from a crystal of pure $\mathrm{La}_{2} \mathrm{CuO}_{4}$, for which we know that the ordered moment in the Neel state is $\sim 0.55 \mu_{B}$, on the same spectrometer under identical conditions. Also, we have made comparisons 
with a vanadium standard and with the weak (004) Bragg reflection in the same crystal for which extinction is not a significant factor. All three comparisons yield consistent results, with the size of the average ordered moment of the SDW equal to $0.15 \pm 0.05 \mu_{B}$ at $T=1.4 \mathrm{~K}$. For the purposes of this estimate, we have assumed a simple model for the SDW order, in the form of magnetic stripes with a width of three spins, separated by non-magnetic anti-phase domain walls. 13 In this case, one requires the presence of two magnetic twin domains of stripes which are oriented at $90^{\circ}$ relative to each other in order to account for the four incommensurate peaks. Even if we use a different model for the spin order, such as a twodimensional square grid of anti-phase domain walls, the variation in the size of the calculated ordered moment is within the error as quoted above. We note that in both such simple candidate models, the predicted third harmonic peaks are extremely weak $(\lesssim 5 \%)$, and in fact we are not able to observe them above the background.

\section{DISCUSSION}

The existence of either short or long-range ordered incommensurate spin density wave peaks now appears to be a common feature of the $\mathrm{La}_{2} \mathrm{CuO}_{4}$-family of superconductors. Such peaks have been observed for several different $\mathrm{Sr}^{2+}$ doping leyels in $\mathrm{Nd}^{3+}$ co-doped samples of $\mathrm{La}_{1.6-x} \mathrm{Nd}_{0.4} \mathrm{Sr}_{x} \mathrm{CuO}_{4} 13$, as well as in purely $\mathrm{Sr}^{2+}$ doped samples of $\mathrm{La}_{2-x} \mathrm{Sr}_{x} \mathrm{CuO}_{4} 20$. Here we have shown that the SDW peaks are clearly seen in oxygen doped samples, as well. For the $\mathrm{La}_{1.6-x} \mathrm{Nd}_{0.4} \mathrm{Sr}_{x} \mathrm{CuO}_{4}$ samples, the SDW ordering and superconductivity coexist but have different transition temperatures with $T_{m}>T_{c}$ for $x=0.12$ and $x=0.15$, and $T_{m} \simeq T_{c}$ for $x=0.20$. For $\mathrm{La}_{2-x} \mathrm{Sr}_{x} \mathrm{CuO}_{4}$, recent experiments suggest that $T_{c}$ $\gtrsim T_{m}$ for $0.10 \lesssim x \lesssim 0.135$ with $T_{c} \simeq T_{m}$ at $x=0.12$. For both of these series of compounds, which involve $\mathrm{Sr}^{2+}$ doping, the quenched randomness of the $\mathrm{Sr}^{2+}$ dopants implies that the hole doping must be fairly homogenous. That is, macroscopic phase separation is prevented by the tendency of the holes to remain in the vicinity of their donor atoms. Hence, it is unlikely that that the magnetic order and the superconductivity occur in macroscopically distinct phase-separated regions of the single crystal samples. In fact, recent $\mu \mathrm{SR}$ and magnetization experiments confirm that the previously studied $\mathrm{La}_{4} \mathrm{~N}_{0.15} \mathrm{CuO}_{4}$ crystal is a bulk superconductor 13.17,30 The coexistence of SDW order and superconductivity for the many superconducting compositions mentioned above argues strongly against their resulting from sample inhomogeneity. Thus, it appears that there is true phase coexistence of superconductivity and longrange incommensurate magnetic order in our $\mathrm{La}_{2} \mathrm{CuO}_{4.12}$ sample. Of course, we cannot rule out phase separation on short length scales, such as into alternating superconducting and SDW lamellae.
The character of the SDW in the $\mathrm{La}_{2} \mathrm{CuO}_{4+y}$ system is clearly of the same fundamental nature as that of its $\mathrm{Sr}^{2+}$-doped brethren, yet is more ideal in several respects. The superconducting $T_{c}$ and SDW ordering $T_{m}$ occur at the same onset temperature $\sim 42 K$ to within the uncertainties, and both temperatures are higher than the highest respective temperatures for $\mathrm{Sr}^{2+}$ doped materials. It had initially been believed that the SDW phase in $\mathrm{La}_{1.6-x} \mathrm{Nd}_{0.4} \mathrm{Sr}_{x} \mathrm{CuO}_{4}$ ordered near $\sim 50 \mathrm{~K}$; however, a subsequent analysis shows that the magnetic order above $30 \mathrm{~K}$ is of short-range, and the ordering below $30 \mathrm{~K}$ is more consistent with glassy behavior.14 As discussed above for our crystal of $\mathrm{La}_{2} \mathrm{CuO}_{4+y}$, we see very similar behavior for incident neutron energies of $5 \mathrm{meV}$ and $13.7 \mathrm{meV}$, which integrate over significantly different energy windows (0.075 versus $0.45 \mathrm{meV}$ HWHM, respectively, with our particular choice for spectrometer collimations). Thus, the magnetic intensity measured throughout the entire temperature range below $\sim 40 \mathrm{~K}$ is predominately static; $\mu \mathrm{SR}$ studies on our samples would determine this more precisely. It is apparent that both $T_{c}$ and $T_{m}$ can be depressed in a particular system by, for example, structural distortions, impurities, or dopant disorder. Although we cannot rule out a coincidence, the ordering of the spin density wave at the same temperature as the superconductivity in our $\mathrm{La}_{2} \mathrm{CuO}_{4+y}$ sample and the observation that both have the highest ordering temperatures for the respective phenomena in $\mathrm{La}_{2} \mathrm{CuO}_{4}$ based materials suggest that the magnetism and the superconductivity are synergistic; further, both are quite sensitive to disorder in the $\mathrm{CuO}_{2}$ planes.

It is now clearly demonstrated that the SDW exists in an orthorhombic crystal structure. In fact, the anisotropy between the in-plane $a$ and $b$ axes is reflected in the ordering pattern of the spins, which have different incommensurabilities $\delta_{H}$ and $\delta_{K}$. Thus as suggested by the previous work of Kimura et al.20 in the $\mathrm{La}_{2-x} \mathrm{Sr}_{x} \mathrm{CuO}_{4}$ system, SDW order in the cuprates is a general phenomenon. The magnitude of the longrange ordered moment at $T=1.4 \mathrm{~K}$ for superconducting $\mathrm{La}_{2} \mathrm{CuO}_{4+y}$ is about $25 \%$ of that in the undoped antiferromagnet $\mathrm{La}_{2} \mathrm{CuO}_{4}$. This is larger than the moment found in the LTT phase of $\mathrm{La}_{1.48} \mathrm{Nd}_{0.4} \mathrm{Sr}_{0.12} \mathrm{CuO}_{4} 13$; therefore, the LTT structure does not enhance the magnetic ordering relative to an orthorhombic structure. Our $\mathrm{La}_{2} \mathrm{CuO}_{4+y}$ sample is to-date the only material in which the magnetic scattering is observed clearly to saturate at low temperature, indicating that the longrange moment is fully ordered. Unlike the situation in the $\mathrm{La}_{1.6-x} \mathrm{Nd}_{0.4} \mathrm{Sr}_{x} \mathrm{CuO}_{4}$ material we cannot yet draw any conclusion regarding possible charge ordering. Scans through the positions of the charge order peaks which were observed in $\mathrm{La}_{1.48} \mathrm{Nd}_{0.4} \mathrm{Sr}_{0.12} \mathrm{CuO}_{4}{ }_{13}$ exhibited a large sloping background in our $\mathrm{La}_{2} \mathrm{CuO}_{4+y}$ sample due to the close proximity of the scattering from the ordered distribution of oxygen interstitial 37 , precluding a firm determination of the existence of charge ordering. We note that since neutrons scatter from small nuclear 
displacements induced by the modulated charge density, the strength of the nuclear superlattice peaks associated with any charge ordering may differ between the LTT and LTO structures, even though the SDW scattering is similar. Preliminary ${ }^{63} \mathrm{Cu}$ NMR results on a small piece of our crystal by Imai and coworkers 35 support an interpretation of the appearance of charge ordering in $\mathrm{La}_{2} \mathrm{CuO}_{4.12}$ below $\sim 55 \mathrm{~K}$ with a temperature dependence similar to that pbserved in $\mathrm{La}_{1.6-x} \mathrm{Nd}_{0.4} \mathrm{Sr}_{x} \mathrm{CuO}_{4}$ by the same method 45 .

The observation of static long range order is interesting in light of the possibility of the existence of a quantum critical point in the cuprate phase diagram. Of course, since true long range order cannot exist at non-zero temperatures in a $2 \mathrm{D}$ system with a continuous symmetry, some spin anisotropy or 3D coupling must be present. In this case, it appears to be the Dzyaloshinskii-Moriya (DM) coupling which breaks the continuous symmetry and makes possible a transition to two-dimensional longrange order at non-zero temperature. However, an algebraic decay state is not ruled out, so long as the spins are correlated over very large distances $(>400 \AA)$. We note that in a model, such as the aforementioned stripe model, with antiphase domain boundaries between ordered spins, the out-of-plane spin canting due to the DM coupling will point in opposite directions on either side of a domain wall. This is a special case of the more general result that due to the DM coupling, an antiferromagnetic SDW with incommensurability $\vec{\delta}$ will give rise to a canted moment with wavevector $-\vec{\delta}$. As a consequence, each $\mathrm{CuO}_{2}$ plane will have no net ferromagnetic moment, con sistent with preliminary magnetization measurements 46 , and in contrast to the yeak ferromagnetic moment observed in pure $\mathrm{La}_{2} \mathrm{CuO}_{4} 42$. For the low-energy inelastic incommensurate scattering, which is believed to be primarily two-dimensional, the $2 \mathrm{meV}$ dynamical correlation length above $T_{m}$ is exceedingly large, $\gtrsim 125 \AA$, which is similar to that measured near the special $x=1 / 8$ doping level in $\mathrm{La}_{2-x} \mathrm{Sr}_{x} \mathrm{CuO}_{4}$ 2. Recently, Aeppli and co-workers have reported pre-divergent behavior in the dynamical properties of $\mathrm{La}_{1.86} \mathrm{Sr}_{0.14} \mathrm{CuO}_{4}$, albeit with a shorter correlation length of $30 \AA$. In this work we clearly see an ordered magnetic phase at an intermediate doping level of the cuprate phase diagram. Even though the spins are correlated three-dimensionally, the fact that the in-plane correlations have long-range order and the between-plane correlations do not, necessitates that the phase transition is driven by the $2 \mathrm{D}$ correlations of a single $\mathrm{CuO}_{2}$ plane. Clearly, studies of the associated 2D critical fluctuations would be most interesting.

The absence of extinction of the $2 \mathrm{meV}$ spin excitations as one cools below $T_{c}$ may now be simply explained. Since our $\mathrm{La}_{2} \mathrm{CuO}_{4+y}$ sample has a long-range ordered ground state, spin wave excitations, which have a temperature independent generalized susceptibility at low $T$, are expected once the sample is cooled below $T_{m}$. This is clearly consistent with the data in Fig. 4(B). In addition,
Kimura et al.20 have shown that $\mathrm{La}_{2-x} \mathrm{Sr}_{x} \mathrm{CuO}_{4}$ with $x=0.15$ does not support a static spin density wave. Again this is consistent with the inelastic scattering data 39 which show a complete gap in the spin excitations at low temperatures for energies $\leq 3.5 \mathrm{meV}$. Our measurements also highlight the need to carry out high resolution measurements of the inelastic magnetic scattering in $\mathrm{La}_{2} \mathrm{CuO}_{4.12}$ to search for dynamic manifestations of the anisotropic incommensurabilities $\delta_{H}$ and $\delta_{K}$ seen in the elastic scattering.

The observed characteristics of the SDW argue against an itinerant electron description of the incommensurate magnetism. Specifically, it is difficult to see how a first principles' delocalized model would predict both interplanar spin correlations and a spin direction which are identical to those in the undoped insulator, $\mathrm{La}_{2} \mathrm{CuO}_{4}$. Also, assuming that the elastic magnetic peaks originate from particle-hole scattering across the Fermi surface of a superconducting condensate, the wavevector should span from node to node of the presumed $d_{x^{2}-y^{2}}$ superconducting order parameter. Such a wavevector would lie along the $(\mathrm{H} 00)$ direction; we find no observable scattering along this direction, but only at the aforementioned incommensurate positions. Therefore, even in the superconductor, the magnetism reflects in detail its Mottinsulator parentage. Additionally, our observations are incompatible with any spiral-type incommensurate modulation of the spin direction.47

A plausible explanation of the SDW peaks is a stripe model similar that proposed to describe $\mathrm{La}_{1.0-x} \mathrm{Nd}_{0.4} \mathrm{Sr}_{x} \mathrm{CuO}_{4} 1013$ and as simulated by Kim et al.48. If stripes are indeed present, then our data impose several important constraints on the model. First, the stripes are not perfectly aligned with the $\mathrm{Cu}-\mathrm{O}-\mathrm{Cu}$ direction, but are slightly rotated. That is, they are tilted by $\sim 3^{\circ}$ from perfect orientational alignment with the underlying spin lattice. This is equivalent to a kink by 1 lattice constant every 17 unit cells. Second, in order to give the observed $L$-dependence, the stripes on neighboring planes must be parallel with each other with the charge domain walls projected nearly on top of each other. This preserves the phase of the 3D spin propagation wavevector. Third, there must exist two types of magnetic twin domains composed of stripes in orthogonal directions with approximately equal populations. Alternatively, a grid model, in which there is a rectangular array of antiphase domain walls running along the orthorhombic directions, is also consistent with the data. Such a model would more naturally account for the differing $\delta_{H}$ and $\delta_{K}$ incommensurabilities by having different domain wall spacings along the two orthorhombic directions. Further experimental investigation of any associated charge ordering is crucial in order to distinguish between these and possibly other models. 


\section{ACKNOWLEDGMENTS}

We gratefully acknowledge G. Aeppli, F.C. Chou, R.J. Christianson, V.J. Emery, H. Fukuyama, M. Greven, K. Hirota, T. Imai, S.A. Kivelson, P.A. Lee, P.K. Mang, A. Tewary, J.M. Tranquada, and B.O. Wells for valuable discussions. The present work was supported by the US-Japan Cooperative Research Program on Neutron Scattering. The work at MIT was supported by the NSF under Grant No. DMR97-04532 and by the MRSEC Program of the National Science Foundation under Award No. DMR98-08941. The work at Tohoku has been supported by a Grant-in-Aid for Scientific Research of Monbusho and the Core Research for Evolutional Science and Technology (CREST) Project sponsered by the Japan Science and Technology Corporation. The work at Brookhaven National Laboratory was carried out under Contract No. DE-AC02-98CH10886, Division of Material Science, U.S. Department of Energy. The work at SPINS is based upon activities supported by the National Science Foundation under Agreement No. DMR-9423101.

${ }^{1}$ M.A. Kastner, R.J. Birgeneau, G. Shirane, and Y. Endoh, Rev. Mod. Phys. 70, 897 (1998).

${ }^{2}$ K. Yamada, C. H. Lee, K. Kurahashi, J. Wada, S. Wakimoto, S. Ueki, H. Kimura, Y. Endoh, S. Hosoya, G. Shirane, R.J. Birgeneau, M. Greven, M.A. Kastner, and Y.J. Kim, Phys. Rev. B 57, 6165 (1998).

${ }^{3}$ B.O. Wells, Y.S. Lee, M.A. Kastner, R.J. Christianson, R.J. Birgeneau, K. Yamada, Y. Endoh, and G. Shirane, Science 277, 1067 (1997).

${ }^{4}$ G. Aeppli, T.E. Mason, S.M. Hayden, H.A. Mook, and J. Kulda, Science 278, 1432 (1997).

${ }^{5}$ H.F. Fong, B. Keimer, D.L. Milius, and I.A. Aksay, Phys. Rev. Lett. 78, 713 (1997); H.F. Fong, B. Keimer, D. Reznik, D.L. Milius, and I.A. Aksay, Phys. Rev. B 54, 6708 (1996).

${ }^{6}$ H.A. Mook, P. Dai, S.M. Hayden, G. Aeppli, T.G. Perring, and F. Dogan, Nature 395, 580 (1998); P. Dai, H.A. Mook, and F. Dogan, Phys. Rev. Lett. 80, 1738 (1998).

${ }^{7}$ N. Bulut, D. Hone, D.J. Scalapino, and N.E. Bickers, Phys. Rev. Lett. 64, 2723 (1990)

${ }^{8}$ Q. Si, Y. Zha, K. Levin, and J.P. Lu, Phys. Rev. B 47, 9055 (1993)

${ }^{9}$ T. Tanamoto, H. Kohno, and H. Fukuyama, J. Phys. Soc. Jpn. 63, 2739 (1994).

10 S.A. Kivelson, E. Fradkin, and V.J. Emery, Nature 393, 550 (1998); V.J. Emery, S.A. Kivelson, and O. Zachar, Phys. Rev. B 56, 6120 (1997).

${ }^{11}$ C. Nayak and F. Wilczek, Phys. Rev. Lett. 78, 2465 (1997).

12 J. Zaanen and O. Gunnarsson, Phys. Rev. B 40, 7391 (1989).

13 J.M. Tranquada, J.D. Axe, N. Ichikawa, A.R. Moodenbaugh, Y. Nakamura, and S. Uchida, Phys. Rev. Lett.
78, 338 (1997); J.M. Tranquada, J.D. Axe, N. Ichikawa, Y. Nakamura, S. Uchida, and B. Nachumi, Phys. Rev. B 54, 7489 (1996); J.M. Tranquada, B.J. Sternlieb, J.D. Axe, Y. Nakamura, and S. Uchida, Nature 375, 561 (1995).

14 J.M. Tranquada, N. Ichikawa, and S. Uchida, Phys. Rev. B 59, 14712 (1999).

${ }^{15}$ A.R. Moodenbaugh, Y. Xu, M. Suenaga, T.J. Folkerts, and R.N. Shelton, Phys. Rev. B 38, 4596 (1988); J.D. Axe, A.H. Moudden, D. Hohlwein, D.E. Cox, K. Mohanty, A.R. Moodenbaugh, and Y. Xu, Phys. Rev. Lett. 62, 2751 (1989).

${ }^{16}$ G.M. Luke, L.P. Le, B.J. Sternlieb, W.D. Wu, Y.J. Uemura, J.H. Brewer, T.M. Riseman, S. Ishibahi, and S. Uchida, Physica C 185-189, 1175 (1991).

${ }^{17}$ B. Nachumi, Y. Fudamoto, A. Keren, K.M. Kojima, M. Larkin, G.M. Luke, J. Merrin, O. Tchernyshyov, Y.J. Uemura, N. Ichikawa, M. Goto, H. Takagi, S. Uchida, M.K. Crawford, E.M. McCarron, D.E. MacLaughlin, and R.H. Heffner, Phys. Rev. B 58, 8760 (1998).

${ }^{18}$ T. Goto, S. Kazama, K. Miyagawa, and T. Fukase, J. Phys. Soc. Jpn. 63, 3494 (1994).

${ }^{19}$ T. Suzuki, T. Goto, K. Chiba, T. Shjinoda, T. Fukase, H. Kimura, K. Yamada, M. Ohashi, and Y. Yamaguchi, Phys. Rev. B 57, 3229 (1998).

${ }^{20}$ H. Kimura, K. Hirota, H. Matsushita, K. Yamada, Y. Endoh, S.H. Lee, C.F. Majkrzak, R.W. Erwin, G. Shirane, M. Greven, Y.S. Lee, M.A. Kastner, and R.J. Birgeneau, Phys. Rev. B 59, 6517 (1999).

${ }^{21}$ H. Kimura et al., unpublished.

${ }^{22}$ H. Takagi, T. Ido, S. Ishibashi, M. Uota, S. Uchida, and Y. Tokura, Phys. Rev. B 40, 2254 (1989)

${ }^{23}$ M. Maki, M. Sera, M. Hiroi, and N. Kobayashi, Phys. Rev. B 53, 11324 (1996).

24 J.C. Grenier, N. Lagueyte, A. Wattiaux, J.P. Doumerc, P. Dordor, J. Etourneau, M. Pouchard, J.B. Goodehough, and J.S. Zhou, Physica C 202, 209 (1992); J.C. Grenier, A. Wattiaux, N. Lagueyte, J.C. Park, E. Marquestaut, J. Etourneau, and M. Pouchard, Physica C 173, 139 (1991).

${ }^{25}$ F.C. Chou, J.H. Cho, and D.C. Johnston, Physica C 197, 303 (1992).

${ }^{26}$ F.C. Chou, D.C. Johnston, S.W. Cheong, and P.C. Canfield, Physica C 216, 66 (1993).

${ }^{27}$ P. Blakeslee, R.J. Birgeneau, F.C. Chou, R.J. Christianson, M.A. Kastner, Y.S. Lee, and B.O. Wells, Phys. Rev. B 57, 13915 (1998).

${ }^{28}$ J.E. Schirber, B. Morosin, R.M. Merill, P.F. Hlava, E.L. Venturini, J.F. Kwak, P.J. Nigrey, R.J. Baughman, and D.S. Ginley, Physica C 152, 121 (1988); G. Demazeau, F. Tresse, Th. Plante, B. Chevalier, J. Etourneau, C. Michel, M. Hervieu, B. Raveau, P. Lejay, A. Sulpice, and R. Tournier, Physica C 153-155, 824 (1988).

${ }^{29}$ H.H. Feng, Z.G. Li, P.H. Hor, S. Bhavaraju, J.F. DiCarlo, and A.J. Jacobson, Phys. Rev. B 51, 16499 (1995).

30 J.E. Ostenson, S. Bud'ko, M. Breitwisch, D.K. Finnemore, N. Ichikawa, and S. Uchida, Phys. Rev. B 56, 2820 (1997).

31 T. Hirayama, M. Nakagawa, A. Sumiyama, and Y. Oda, Phys. Rev. B 58, 5856 (1998).

32 Z.G. Li, H.H. Feng, Z.Y. Yang, A. Hamed, S.T. Ting, P.H. Hor, S. Bhavaraju, J.F. DiCarlo, and A.J. Jacobson, 
Phys. Rev. Lett. 77, 5413 (1996).

${ }^{33}$ D.C. Johnston, Phys. Rev. Lett. 62, 957 (1989)

34 T. Nakano, M. Oda, C. Manabe, N. Momono, Y. Miura, and M. Ido, Phys. Rev. B 49, 16000 (1994).

${ }^{35}$ T. Imai et al., unpublished.

${ }^{36}$ B.O. Wells, R.J. Birgeneau, F.C. Chou, Y. Endoh, D.C. Johnston, M.A. Kastner, Y.S. Lee, G. Shirane, J.M. Tranquada, and K. Yamada, Z. Phys. B 100, 535 (1996).

37 Y.S. Lee et al., unpublished.

${ }^{38}$ P.G. Radaelli, J.D. Jorgenson, A.J. Shultz, B.A. Hunter, J.L. Wagner, F.C. Chou, and D.C. Johnston, Phys. Rev. B 49, 6239 (1994).

${ }^{39}$ K. Yamada, S. Wakimoto, G. Shirane, C.H. Lee, M.A. Kastner, S. Hosoya, M. Greven, Y. Endoh, and R.J. Birgeneau, Phys. Rev. Lett. 75, 1626 (1995).

${ }^{40}$ T. Fretloft, G. Shirane, S. Mitsuda, J.P. Remeika, and A.S. Cooper, Phys. Rev. B 37, 137 (1988).

${ }^{41}$ D. Vaknin, S.K. Sinha, D.E. Moncton, D.C. Johnston, J.M. Newsam, C.R. Safinya, and H.E. King, Jr., Phys. Rev. Lett. 58, 2802 (1987).

${ }^{42}$ T. Thio, T.R. Thurston, N.W. Preyer, P.J. Picone, M.A. Kastner, H.P. Jenssen, D.R. Gabbe, C.Y. Chen, R.J. Biregeneau, A. Aharony, Phys. Rev. B 38, 905 (1988); T. Thio, C.Y. Chen, B.S. Freer, D.R. Gabbe, H.P. Jenssen, M.A. Kastner, P.J. Picone, N.W. Preyer, and R.J. Birgeneau, Phys. Rev. B 41, 231 (1990).

${ }^{43}$ S. Shamoto, M. Sato, J.M. Tranquada, B.J. Sternlieb, and G. Shirane, Phys. Rev. B 48, 13817 (1993).

${ }^{44}$ M. v. Zimmermann, A. Vigliante, T. Niemöller, N. Ichikawa, T. Frello, J. Madsen, P. Wochner, S. Uchida, N.H. Andersen, J.M. Tranquada, D. Gibbs, and J.R. Schneider, Europhys. Lett. 41, 629 (1998).

${ }^{45}$ A.W. Hunt, P.M. Singer, K.R. Thurber, and T .Imai, Phys. Rev. Lett. 82, 4300 (1999).

${ }^{46}$ J.K. Thomas et al., unpublished.

47 B.I. Shraiman and E.D. Siggia, Phys. Rev. Lett. 62, 1564 (1989); B.I. Shraiman and E.D. Siggia, Phys. Rev. B 42, 2485 (1990);

${ }^{48}$ Y.J. Kim, R.J. Birgeneau, M.A Kastner, Y.S. Lee, Y. Endoh, G. Shirane, and K. Yamada, cond-mat/9902248,

Phys. Rev. B (01Aug99). 